TITLE:

\title{
Priming and implicit recognition depend on similar temporal changes in perceptual representations.
}

\author{
AUTHOR(S):
}

Miyoshi, Kiyofumi; Ashida, Hiroshi

\section{CITATION:}

Miyoshi, Kiyofumi ...[et al]. Priming and implicit recognition depend on similar temporal changes in perceptual representations.. Acta psychologica 2014, 148: 6-11

\section{ISSUE DATE:}

2014-01-28

URL:

http://hdl.handle.net/2433/182954

\section{RIGHT:}

@ 2014 Elsevier B.V.; この論文は出版社版でありません。引用の際には 出版社版をご確認ご利用ください。; This is not the published version. Please cite only the published version. 
Priming and implicit recognition depend on similar temporal changes in perceptual representations

Kiyofumi MIYOSHI

Graduate School of Letters, Kyoto University, Sakyo, Kyoto 6068501, Japan

TEL: $+81-75-753-2753$

E-mail:miyoshi80@gmail.com

Hiroshi ASHIDA

Graduate School of Letters, Kyoto University, Sakyo, Kyoto 6068501, Japan TEL: +81-75-753-2753

E-mail: ashida@psy.bun.kyoto-u.ac.jp

Corresponding author

Kiyofumi MIYOSHI

TEL: +81-75-753-2753

E-mail:miyoshi80@gmail.com 


\section{Abstract}

Previous studies have reported that longer stimulus presentation decreases the magnitude of priming. In the present study, we used meaningless kaleidoscope images, which were reported to minimize conceptual processing, to investigate the mechanism of the phenomenon. We assessed the impact of stimulus duration on perceptual priming (Experiment 1) and implicit recognition memory (Experiment 2). Both the magnitude of priming and the accuracy of implicit recognition were lower with the longer stimulus presentation $(350 \mathrm{~ms})$ than with the shorter presentation $(250 \mathrm{~ms})$. This coincidence of temporal dynamics between priming and implicit recognition suggests similar underlying memory mechanisms. In both cases, the decrease of performance with longer presentation can be explained by either changes in perceptual processes or interference from explicit memory retrieval.

Key words: priming, implicit memory, recognition memory, implicit recognition 


\section{Introduction}

\section{1. "Rise and fall" pattern of priming}

Priming refers to the retrieval of pre-acquired representations without a need for conscious awareness of memory (Graf \& Schacter, 1985). Furthermore, the magnitude of priming is often assessed by behavioral measures such as faster response time or greater accuracy. Several studies suggest that short presentation of a previous stimulus provides positive priming, whereas longer presentation leads to weaker or even negative priming (Barbot \& Kouider, 2012; Faivre \& Kouider, 2011; Huber \& O’Reilly, 2003; Zago, Fenske, Aminoff, \& Bar, 2005). Among these previous studies, Zago et al. (2005) is unique in its examination of the "rise and fall" pattern in a fine timescale. They presented color photographs of familiar objects, and participants identified the objects as natural or manmade. The magnitude of priming increased with short presentation of primes (40-250 ms) but decreased with longer presentation (350-1900 ms). The researchers observed that the rise and fall pattern was present with the maximal magnitude of priming for $250 \mathrm{~ms}$ of previous stimulus exposure.

They explained this pattern by introducing a combined model of "sharpening" (Desimone, 1996) and "selection" (Wiggs \& Martin, 1998). According to this model, short presentation of a previous stimulus improves the coding of stimulus features and sharpens object representation. Longer presentation, however, elicits a selection process based on high-level information and semantic knowledge. Selection leads to the continued representation of the features essential for identifying the object and the discarding of non-essential features. Therefore, overlapping features between the representation of the previous stimulus and the target decrease, which reduces the 
magnitude of priming.

Although the combined model of sharpening and selection explains the rise and fall pattern, it is only one possible explanation, and it requires validation as well as allows for other accounts. Sharpening is supported by physiological evidence which indicates that neurons in the inferior temporal cortex of macaque monkeys represent only global properties of stimuli at $\sim 130 \mathrm{~ms}$, before becoming more stimulus-specific at $\sim 240 \mathrm{~ms}$ (Tamura \& Tanaka, 2001). It is, however, not as well supported that selection leads to the "fall" pattern of priming, because selection is considered to cause a faster and more efficient behavioral response (Wiggs \& Martin, 1998). Considering that the task requirement in Zago et al. (2005) was to judge whether the objects were natural or manmade, selection of the essential features for identifying a certain object should have been highly beneficial, and we could argue that selection should have increased rather than decreased the magnitude of priming. Therefore, we need an alternative account for the fall of priming.

We must also note that perceptual priming and conceptual priming might have co-occurred in Zago et al. (2005), given the properties of their task and stimuli. Considering that perceptual and conceptual priming have different attributes and depend on distinct neural mechanisms (Voss, Schendan, \& Paller, 2010), research must identify the factors involved in forming the rise and fall pattern.

To address the aforementioned issues, we examined the rise and fall pattern of priming under conditions in which conceptual processes (selection and conceptual priming) are not likely to arise. An effective method of excluding the influences of conceptual processes is to use stimuli without pre-existing memory (Deschepper \& Treisman, 1996; Musen \& Treisman, 1990; Schacter, Cooper, \& Delaney, 1990; Voss \& Paller, 2010a). We 
used the meaningless kaleidoscope images introduced by Voss and colleagues (Voss, Baym, \& Paller, 2008; Voss \& Paller, 2010a), and employed a color-decision task similar to that in their studies. In the present study's Experiment 1, participants judged the number of colors in minimally meaningful and difficult-to-recognize kaleidoscope images. If the rise and fall pattern is replicated under the conditions of the present experiment, the fall cannot be explained by selection with higher-level semantic information.

\subsection{Implicit memory processes in recognition memory tests}

Another question is whether the rise and fall of performance is specific to priming or more generally observed for implicit memory. To investigate this question and elucidate the underlying mechanism, we compared the impact of stimulus duration on priming in Experiment 1 with that on the recently reported phenomenon of "implicit recognition" in Experiment 2.

One of the most fundamental topics in the study of memory is the dissociation between implicit and explicit memory (Graf \& Schacter, 1985; Schacter \& Tulving, 1994). Most studies have attempted to demonstrate one-to-one correspondence between performances of a certain task and a certain memory (Berry, Shanks, \& Henson, 2008). In this view, the priming task is considered to be driven only by implicit memory and the recognition task only by explicit memory.

However, several studies suggest that recognition judgments might be affected by implicit memory (Jacoby \& Whitehouse, 1989; Rajaram, 1993; Tunney \& Fernie, 2007). In these studies, target stimuli primed by a masked preceding stimulus were likely to be recognized as old. Furthermore, recent studies have more clearly demonstrated the 
contribution of implicit memory to recognition memory performance (Vargas, Voss, \& Paller, 2012; Voss et al., 2008; Voss \& Paller, 2009; Voss \& Paller, 2010b). In Voss and Paller (2009), participants studied kaleidoscope images in either a full- or divided-attention condition. In a forced-choice recognition test, participants selected the old image and reported their awareness of memory via a remember/know/guess procedure. Surprisingly, performance was better under the divided-attention condition than under the full-attention condition. Moreover, accurate recognition occurred without conscious awareness of memory; the accuracy of the "guess" response was very high and was even higher than that of the "know" response. This implicit recognition was associated with the N300 effect in the evoked potentials; it was similar to that associated with perceptual priming and was distinct from the positive brain potential associated with recollection and familiarity. These results supplement their argument that implicit recognition and priming depend on similar implicit memory mechanisms.

These findings indicate a crucial contribution of implicit memory processes to recognition performance. This implicit recognition, however, is highly elusive, difficult to replicate (Jeneson, Kirwan, \& Squire, 2010), and demands many strict prerequisites (Voss \& Paller, 2010b). Furthermore, there is little behavioral evidence indicating that implicit recognition relies on implicit memory processes; existing evidence merely suggests that lateralized visual fluency may be responsible for implicit recognition (Vargas et al., 2012).

In Experiment 2, to obtain insights into possible common mechanisms, we assessed whether implicit recognition exhibits dependence that is similar to that of priming on stimulus duration. As previously mentioned, the magnitude of priming rises and falls with stimulus duration. Therefore, if implicit recognition and priming are based on 
similar implicit memory processes, the performance of implicit recognition would vary following the same time course as that of the magnitude of priming. Using kaleidoscope images similar to those in Experiment 1, Experiment 2 investigated whether implicit recognition shows the fall pattern, because we consider this unique and counterintuitive fall pattern as the most characteristic feature of the temporal dynamics of priming.

\section{Experiment 1}

We investigated the time course of priming using meaningless kaleidoscope images. Because of the small number of available images, we assessed the "rise" and "fall" for different groups of participants (referred to as the "brief" and "long" conditions, respectively).

\subsection{Materials and Methods}

\subsubsection{Participants}

A total of 32 students from Kyoto University volunteered to participate in Experiment 1: 16 (11 men, 5 women; ages 18-24 years) participated in the brief condition and the remaining 16 (12 men, 4 women; ages 19-28 years) participated in the long condition. They were paid according to the Kyoto University standard. All participants had normal color vision.

\subsubsection{Materials and Procedure}

Courtesy of Dr. Voss, we used 180 kaleidoscope images introduced in Voss et al. (2008) and Voss and Paller (2010a). Among these images, 90 contained three colors and the 
remaining contained four or five colors. Images were randomly assigned to experimental blocks for each participant and were displayed on a dark background on a computer monitor (23" Apple Cinema Display) using the software Presentation (Neurobehavioral Systems).

Fig. 1 illustrates the image sequence in the brief condition. Participants were tested for 10 blocks of prime-test sessions. In each prime session, 12 images were presented in random order and were immediately followed by a mask. The mask was a random color image generated by the Texture-inf software (http://www.geocities.jp/iooiau/textureinf.html). Half the kaleidoscope images were presented for $150 \mathrm{~ms}$ and the remaining were presented for $250 \mathrm{~ms}$. The duration of the mask was either 350 or $250 \mathrm{~ms}$, ensuring that the total duration of each trial was 500 ms. These two types of trials were alternately presented, and the type of the first trial in each prime session was counterbalanced across blocks. Inter-trial intervals were 2000 ms, wherein participants pressed a button to indicate whether the previously presented image contained three colors. The test session followed the prime session after a $15^{\text {-s }}$ break. Six 150-ms-primed images, six 250-ms-primed images, and six novel images were presented for $2000 \mathrm{~ms}$ in random order. Participants performed the same task as in the prime session as quickly and as accurately as possible before the image disappeared. Inter-trial intervals were $2000 \mathrm{~ms}$.

In the long condition, participants performed the same color-decision task as in the brief condition, except that stimulus durations in the prime session were $250 \mathrm{~ms}$ and $350 \mathrm{~ms}$, with mask durations being $250 \mathrm{~ms}$ and $150 \mathrm{~ms}$, respectively. 


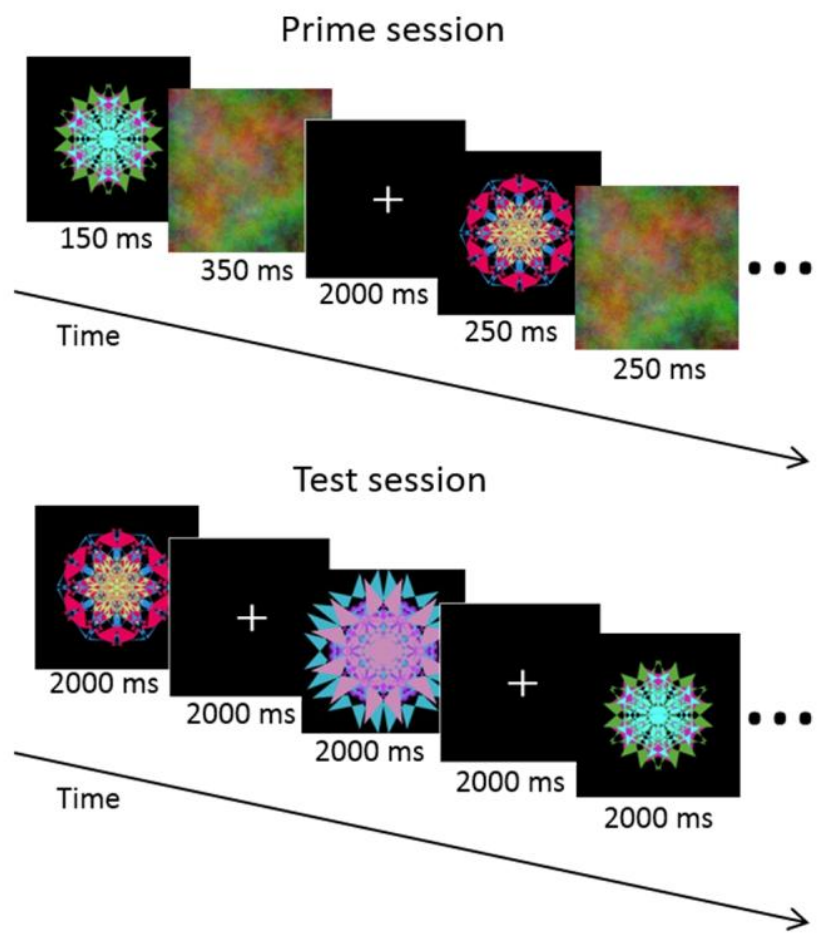

Fig. 1. Trial presentation sequence in the brief condition. In the prime sessions, kaleidoscope images were alternately presented for either 150 or $250 \mathrm{~ms}$. In the test sessions images were presented for $2000 \mathrm{~ms}$. Participants judged whether each image contained three colors.

\subsection{Results and Discussion}

Table 1 reports mean reaction times and response accuracies recorded in the test sessions. Analyses included only trials with correct responses. The magnitude of priming was defined as the difference between mean reaction times in novel and primed trials (Fig. 2). We conducted a two-way mixed design ANOVA on the mean magnitude of priming with condition (brief/long) as a between-participant factor and stimulus duration (shorter/longer) as a within-participant factor. There was no significant main 
effect of condition $(F(1,30)=.63, p=.44)$ or stimulus duration $(F(1,30)=.77, p=.39)$; importantly, there was a significant interaction $(F(1,30)=10.05, p=.004)$. A simple main effects analysis revealed that priming was greater for longer presentation in the brief condition $(F(1,30)=4.40, p=.04)$, whereas it was greater for shorter presentation in the long condition $(F(1,30)=8.19, p=.008)$.

In addition, we calculated the difference between the mean accuracy in novel and primed trials in each condition. Then, we conducted a two-way mixed design ANOVA with condition (brief/long) as a between-participant factor and stimulus duration (shorter/longer) as a within-participant factor. There was no significant main effect of condition $(F(1,30)=.27, p=.61)$ or stimulus duration $(F(1,30)=.01, p=.92)$, nor was there significant interaction $(F(1,30)=.01, p=.92)$, indicating that the difference in the magnitude of priming depicted in Fig. 2 is not due to the speed-accuracy trade-off.

Although different groups of participants were involved in the different conditions, the performances in the $250-\mathrm{ms}$ conditions did not significantly differ between the groups $(t=.52, p=.61$ for RT and $t=.72, p=.48$ for accuracy).

To summarize Experiment 1, the rise and fall pattern of priming was successfully replicated when the influence of conceptual processes (selection and conceptual priming) was unlikely to contribute. One could posit a possible confounding effect of the different mask durations for each prime duration, for the constant total of $500 \mathrm{~ms}$. We believe, however, that this should not be a crucial issue, at least for the fall of priming. If the mask impaired priming, the magnitude of priming should have been the greatest for 350-ms-primed trials, in which the mask was the shortest. However, this was not the case. The rise could be affected, but it is unlikely that the primes are sufficiently identified immediately after the onset, and the rise of priming should be expected 
regardless.

We should note that $2000 \mathrm{~ms}$ of kaleidoscope presentation led to significant priming in Voss and Paller (2010a), whereas $350 \mathrm{~ms}$ of presentation did not do so in the present study. Unlike in our study, they introduced an auditory 1-back task as a distractor in the study sessions, which we consider to cause the discrepancy. The general discussion will further discuss this point.

Table 1. Mean reaction times and mean response accuracies for each condition.

\begin{tabular}{lllll}
\hline & novel trials & 150-ms-primed trials & 250-ms-primed trials & 350 -ms-primed trials \\
\hline Brief condition & & & & \\
Reaction time & $1126(181)$ & $1107(194)$ & $1085(185)$ & \\
Response accuracy & $.77(.09)$ & $.77(.09)$ & $.76(.09)$ & \\
Long condition & & & $1049(207)$ & $1087(228)$ \\
Reaction time & $1083(212)$ & & $.74(.10)$ & $.74(.06)$ \\
Response accuracy & $.73(.08)$ & & \\
\hline
\end{tabular}

SD in parentheses

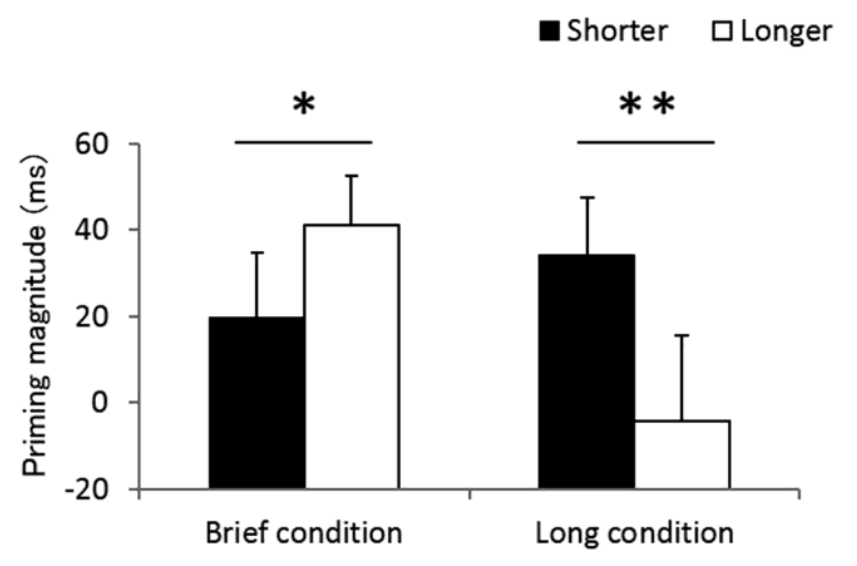

Fig. 2. Magnitude of priming for shorter $(150 \mathrm{~ms}$ in the brief condition and 250 $\mathrm{ms}$ in the long condition) and longer (250 $\mathrm{ms}$ in the brief condition and $350 \mathrm{~ms}$ in the long condition) presentation. Error bars indicate the standard error of the mean. * indicates $p<.05$ and ** indicates $p<.01$. 


\section{Experiment 2}

We investigated the impact of stimulus duration on the performance of implicit recognition. If implicit recognition and priming are based on similar implicit memory processes, the performance of implicit recognition should exhibit a fall pattern similar to that in Experiment 1. We investigated only the fall pattern because we consider this counterintuitive pattern as the most characteristic feature of the temporal changes in implicit memory processes, whereas the rise is naturally expected.

\subsection{Materials and Methods}

\subsubsection{Participants}

Twelve students (8 men, 4 women; ages 18-25 years) at Kyoto University volunteered to participate in Experiment 2. They were paid according to the Kyoto University standard. All participants had normal color vision.

\subsubsection{Materials and Procedure}

The stimuli were 396 kaleidoscope images used in Voss et al. (2008), all containing three colors. They were similar to the images used in Experiment 1 . There were 180 visually similar pairs and 36 other images used as primacy and recency buffers in the study sessions. Ten pairs and two buffers were used in each study-test block. Images were randomly assigned to experimental blocks for each participant and were displayed on a dark background on a computer monitor (23" Apple Cinema Display) using the software Presentation (Neurobehavioral Systems).

Fig. 3 illustrates the Experiment 2 trial sequence. Participants studied the 
kaleidoscope images and distinguished between old images and novel images in the test session. Participants were tested for 18 study-test blocks.

In the study session, 12 images were presented (one from each of the 10 pairs and two buffers). Half the images were presented for $250 \mathrm{~ms}$ and the other half for $350 \mathrm{~ms}$. The two durations of trials were alternately presented, and the duration of the first trial was counterbalanced across blocks. Images were immediately followed by a mask, and there were two mask durations (250 and $150 \mathrm{~ms}$ ) to standardize the total duration of presentations at $500 \mathrm{~ms}$.

The first and last images in the study sessions were used as primacy and recency buffers and were not included in the test session. To inhibit semantic encoding, we used articulatory suppression (Murray, 1967). Participants explicitly encoded the images while continuously vocalizing the word "the" approximately once per second during the study session. There was no guide for the timing of vocalization. Inter-trial interval was $2000 \mathrm{~ms}$.

In the test session, $15 \mathrm{~s}$ after the study session, forced-choice recognition tests were conducted. Ten images (excluding the first and last buffer images) from the study session were tested in random order. In each trial, a pair of images (a studied image and its visually similar foil) appeared alongside each other for $2000 \mathrm{~ms}$. The studied image was equally likely to appear on the left or right. During the following $2500 \mathrm{~ms}$, participants indicated the side on which the studied image was presented by pressing a key. Participants were then asked to report their awareness of memory (metamemory) via a remember/know/guess procedure. A remember response indicated that the recognition was accompanied by high confidence and specific details about the kaleidoscope image. A know response indicated that the recognition was supported by a 
vague familiarity but no details. A guess response suggested that participants had no awareness of memory retrieval and no feeling of familiarity. Participants were told not to hesitate to guess. Inter-trial intervals were $2000 \mathrm{~ms}$.

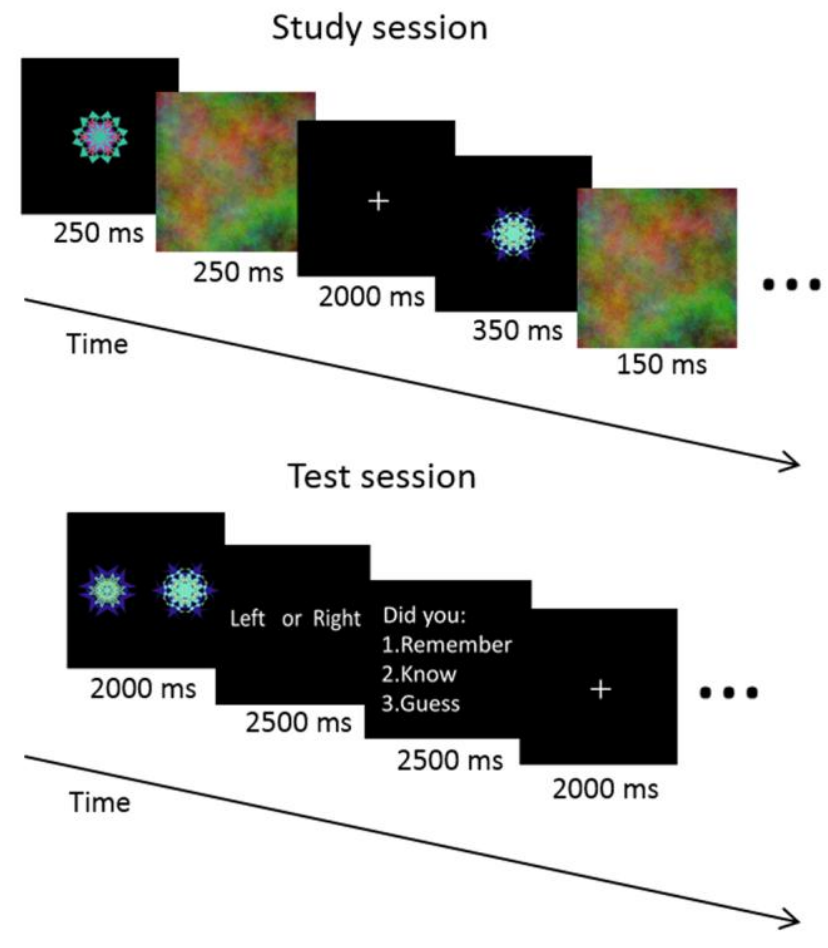

Fig. 3. Trial presentation sequence in Experiment 2. In the study sessions, participants alternately encoded kaleidoscope images for 250 and $350 \mathrm{~ms}$ while continuously vocalizing the word "the." In the test sessions, participants discriminated between old and novel images and reported their awareness of memory.

\subsection{Results and Discussion}

We predicted that the guess responses (implicit recognition) would be more accurate for the images that were previously presented for $250 \mathrm{~ms}$ than for those presented for $350 \mathrm{~ms}$. 
Fig. 4A depicts metamemory response rates. A two-way repeated measures ANOVA was conducted with metamemory category and stimulus duration as within-participant factors. There was a significant main effect of metamemory category $(F(2,22)=9.93, p$ $<.001)$ but no significant main effect of stimulus duration $(F(1,11)=0.00, p>.99)$. In addition, there was no significant interaction $(F(2,22)=2.35, p=.12)$, indicating that the difference in stimulus durations did not impact metamemory judgments. This outcome demonstrates that difference between the accuracy rates in different conditions cannot be ascribed to the shifts of criteria in metamemory judgments.

The mean proportion of correct responses for each metamemory category is shown in Fig. 4B. A repeated measures ANOVA was conducted with metamemory category and stimulus duration as within-participant factors. There was a significant main effect of metamemory category $(F(2,22)=28.65, p<.001)$ but no significant main effect of stimulus duration $(F(1,11)=.87, p=.37)$; importantly, there was a significant interaction between stimulus duration and metamemory category $(F(2,22)=3.67, p$ $=.04)$. A simple main effects analysis revealed that the mean proportion of correct responses for $250-\mathrm{ms}^{-}$-studied images was higher than that for $350-\mathrm{ms}^{-}$studied images for the guess response $(F(1,33)=4.57, p=.04)$, but it revealed no significant difference for the know response $(F(1,33)=1.08, p=.31)$ or the remember response $(F(1,33)=$ 1.94, $p=.17$ ). Moreover, a one-sample t-test revealed that the mean proportion of correct responses for 250 - $\mathrm{ms}^{-}$studied images in the guess category was significantly above the chance level $(t(11)=3.15, p=.01)$, whereas that for 350-ms-studied images was not $(t(11)=-.44, p=.67)$. These results suggest that accurate recognition without awareness of memory occurred only for the 250-ms-studied images.

To summarize the results, implicit recognition was present only for the 250 -ms-studied 
images, and, importantly, implicit recognition performance declined at the same time point as did the magnitude of priming in Experiment 1. Thus, the results demonstrate the first behavioral evidence that implicit recognition and priming exhibit a similar pattern of temporal dynamics, suggesting similar implicit memory mechanisms underlying the two phenomena.

Although the accuracy of the guess response varied significantly with the difference in stimulus durations, that of the remember and know responses did not. This distinction is consistent with the EEG result in Voss and Paller (2009). In their study, only the guess responses were associated with negative brain potentials (N300); the remember and know responses were associated with positive potentials (P200 and LPC). These results indicate that only the guess responses rely strongly on implicit memory processes, whereas the remember and know responses may primarily depend on explicit retrieval processes.

However, Fig. 4B indicates that the mean proportion correct of the guess and know responses were higher for $250{ }^{-\mathrm{ms}^{-}}$studied images than for $3500^{-} \mathrm{ms}^{-}$studied images. On the other hand, the mean proportion correct of the remember responses reflected the opposite pattern, although the differences in the know and remember responses were not significant. This outcome suggests that the know responses as well as the guess responses to some extent depend on implicit memory processes. Given that Voss and Paller (2009) reported a clear distinction in EEG results between remember/know and guess, the metamemory judgment criteria may differ slightly between our study and theirs. Participants could have been more dependent on implicit memory processes for the know response in the present study than in their study.

Finally, we note that $2000 \mathrm{~ms}$ of stimulus presentation led to robust implicit 
recognition in Voss and Paller (2009), but $350 \mathrm{~ms}$ of presentation in the present study did not. We address this discrepancy in the general discussion.

A

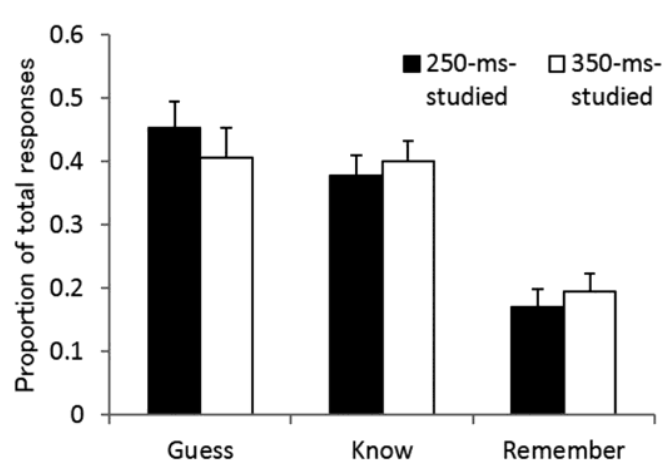

B

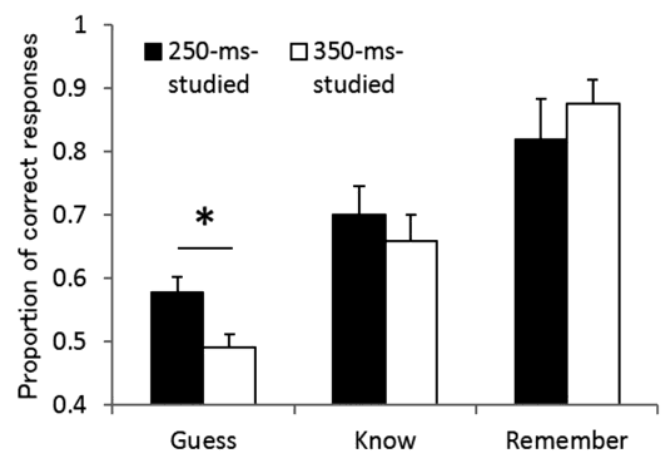

Fig. 4. Results of Experiment 2. (A) The mean proportion total responses for each metamemory category. (B) The mean proportion correct for guess, know, and remember responses. Error bars indicate the standard error of the mean. * indicates $p<.05$.

\section{General discussion}

\subsection{Possible mechanism of the rise and fall: bottom-up changes in perceptual processes}

We observed the rise and fall of priming in Experiment 1 and the fall of implicit recognition in Experiment 2. The results suggest similar implicit memory mechanisms underlying these phenomena.

Bar (2001) and Zago et al. (2005) suggest that the magnitude of priming reflects the state of pre-acquired representations of a previous stimulus. Therefore, perceptual representations might reach optimum efficiency at approximately $250 \mathrm{~ms}$ of previous stimulus exposure, which explains the rise pattern. This process can be labeled as "sharpening" (Zago et al., 2005). However, considering that the target images were 
meaningless and participants did not have pre-existing memory of them, the fall pattern observed in the present study is difficult to explain by "selection" (Zago et al., 2005). Instead, we propose that the bottom-up changes in visual perception cause the rise and fall. Perceptual representations reach optimum efficiency at a certain amount of stimulus exposure and are less efficient thereafter because of bottom-up changes in perceptual processes.

Experiment 1 also found that priming almost completely disappeared when prime stimuli were presented for $350 \mathrm{~ms}$. Many other studies, using more meaningful stimuli, demonstrated significant priming for longer primes (McAuliffe \& Knowlton, 2009; Voss \& Gonsalves, 2010; Zago et al., 2005). Thus, the results suggest that the efficiency of perceptual representations may disappear during the early period of stimulus presentation and that only more durable conceptual representations may endure. Consistent with this view, several studies have proposed that representations at lower levels might be activated and habituated more quickly than those at higher levels (Huber, Shiffrin, Lyle, \& Ruys, 2001; Huber \& O’Reilly, 2003). It is more economical to code abstracted and conceptualized information of objects than to maintain codes for their direct physical features.

\subsection{Alternative account: explicit memory interference}

Another possible cause of the rise and fall is interference by explicit memory retrieval. First, identifying the stimulus requires a certain duration. Second, viewing the stimulus after identifying it enables additional explicit memory encoding. Explicit retrieval during the test might reduce the extent to which implicit memory signals determine the decision and impair implicit memory performance. 
This reasoning effectively explains the discrepancies between our study and previous studies. We have noted that $2000 \mathrm{~ms}$ of stimulus presentation led to robust priming or implicit recognition in previous studies (Voss \& Paller, 2009; Voss \& Paller, 2010a), whereas $350 \mathrm{~ms}$ of presentation did not do so in the present study. The major difference between our study and theirs is that unlike us, they introduced an auditory 1-back task as a distractor in the study sessions. This distractor might impair elaborative encoding during the study phase. Therefore, with a demanding secondary task, explicit memory could be effectively suppressed even for the 2000-ms stimulus exposure, thus not allowing it to interfere with later implicit memory. Thus, the difference between our results and those of previous studies might indirectly support the "explicit memory interference" explanation of the fall pattern.

In addition, the performance of implicit recognition in our experiment was relatively low. The accuracy of the guess responses for 250-ms-studied images in the present study was 57.8\%, whereas in Voss and Paller (2009), the accuracy of the same in the divided-attention condition was approximately $78 \%$, higher than the accuracy of the know responses. Participants may have elaborately encoded kaleidoscopes without the demanding 1-back task in the present study, which might have reduced the contribution of implicit memory to recognition performance, thus decreasing implicit recognition performance. In fact, Voss and Paller (2009) reported that implicit recognition performance declined when the participants did not perform the 1-back task during the study.

Furthermore, Lee, Blumenfeld, and D'Esposito (2013) reported that applying transcranial magnetic stimulation over the dorsolateral prefrontal cortex (DLPFC) improved the accuracy of implicit recognition and suggested that explicit memory 
processes mediated by the DLPFC can interfere with implicit recognition. These results indirectly support the "explicit memory interference" explanation of the fall of implicit memory.

\subsection{Implicit memory processes in various types of memory tests}

The present study demonstrates that recognition memory can be driven by both implicit and explicit retrievals, which raises doubts about the one-to-one correspondence between a certain task and a certain memory. We consider that, in any type of memory task, people acquire many levels of representations from stimulus presentation, and the contribution of these representations to performance may vary in relation to the task's attributes or the participants' strategy. Turk-Browne, Yi, and Chun (2006) also posited that implicit and explicit memory can rely on the same encoding factors and similar perceptual representations. Further research is necessary to arrive at a firm conclusion, but researchers should remain aware of the possibility of multiple factors even when using a single memory task.

\section{Acknowledgments}

We thank Dr. Joel Voss for providing stimulus images and valuable advice. This work was supported by a JSPS Grant-in-Aid for scientific research (S) \# 22220003. 


\section{References}

Bar M. (2001). Viewpoint dependency in visual object recognition does not necessarily imply viewer-centered representation. Journal of Cognitive Neuroscience, 13, 793-799.

Barbot, A., \& Kouider, S. (2012). Longer is not better: nonconscious overstimulation reversespriming influences under interocular suppression. Attention, Perception, \& Psychophysics, 74, 174-184.

Berry, C. J., Shanks, D. R., \& Henson, R. N. (2008). A unitary signal-detection model of implicit and explicit memory. Trends in Cognitive Science, 12, 367-373.

DeSchepper, B., \& Treisman, A. (1996). Visual memory for novel shapes: implicit coding without attention. Journal of Experimental Psychology. Learning, Memory, and Cognition, 22, 27-47.

Desimone, R. (1996). Neural mechanisms for visual memory and their role in attention. Proceedings of the National Academy of Sciences of the United States of America, 93, 13494-13499.

Faivre, N., \& Kouider, S. (2011). Increased sensory evidence reverses nonconscious priming during crowding. Journal of Vision, 11, 1-13.

Graf, P., \& Schacter, D. L. (1985). Implicit and explicit memory for new associations in normal and amnesic subjects. Journal of Experimental Psychology. Learning, Memory, and Cognition, 11, 501-518.

Huber, D. E., \& O'Reilly, R. C. (2003). Persistence and accommodation in short-term 
priming and other perceptual paradigms: Temporal segregation through synaptic depression. Cognitive Science, 27, 403-430.

Huber, D. E., Shiffrin, R. M., Lyle, K. B., \& Ruys, K. I. (2001). Perception and preference in short-term word priming. Psychological Review, 108, 149-182.

Jacoby, L. L., \& Whitehouse, K. (1989). An illusion of memory: false recognition influenced by unconscious perception. Journal of Experimental Psychology: General, 118, 126-135.

Jeneson, A., Kirwan, C. B., \& Squire, L. R. (2010). Recognition without awareness: An elusive phenomenon. Learning \& Memory, 17, 454-459.

Lee, T. G., Blumenfeld, R. S., D’Esposito, M. (2013). Disruption of dorsolateral but not ventrolateral prefrontal cortex improves unconscious perceptual memories. Journal of neuroscience, 33, 13233-13237

McAuliffe, S. P., \& Knowlton, B. J. (2009). The time course of object encoding. Acta Psycholgica, 132, 213-220.

Murray, D. J. (1967). The role of speech responses in short-term memory. Canadian Journal of Psychology, 21, 263-276.

Musen, G., \& Treisman, A. (1990). Implicit and explicit memory for visual patterns. Journal of Experimental Psychology. Learning, Memory, and Cognition, 16, 127-137.

Rajaram, S. (1993). Remembering and knowing: two means of access to the personal past. Memory \& Cognition, 21, 89-102.

Schacter, D. L., Cooper, L. A., \& Delaney, S. M. (1990). Implicit memory for unfamiliar objects depends on access to structural descriptions. Journal of Experimental Psychology: General, 119, 5-24. 
Schacter, D.L., \& Tulving, E. (1994). What are the memory systems of 1994? In Memory Systems 1994, D.L. Schacter and E. Tulving, eds. (Cambridge, MA: The MIT Press), pp. 1-38.

Tamura, H., \& Tanaka, K. (2001). Visual response properties of cells in the ventral and dorsal parts of the macaque inferotemporal cortex. Cerebral Cortex, 11, 384-399.

Tunney, R. J., \& Fernie, G. (2007). Repetition priming affects guessing not familiarity. Behavioral and Brain Functions, 3, 40-46.

Turk-Browne, N. B., Yi, D. J., \& Chun, M. M. (2006). Linking implicit and explicit memory: common encoding factors and shared representations. Neuron, 49, 917-927.

Vargas, I. M., Voss, J. L., \& Paller, K. A. (2012). Implicit recognition based on lateralized perceptual fluency. Brain Sciences, 2, 22-32.

Voss, J. L., Baym, C. L., \& Paller, K. A. (2008). Accurate forced-choice recognition without awareness of memory retrieval. Learning \& Memory, 15, 454-459.

Voss, J. L. \& Gonsalves, B. D. (2010). Time to go our separate ways: Opposite effects of study duration on priming and recognition reveal distinct neural substrates. Frontiers in Human Neuroscience, 4, 227.

Voss, J. L., \& Paller, K. A. (2009). An electrophysiological signature of unconscious recognition memory. Nature Neuroscience, 12, 349-355.

Voss, J. L., \& Paller, K. A. (2010a). Real-time neural signals of perceptual priming with unfamiliar geometric shapes. The Journal of Neuroscience, 30, 9181-9188.

Voss, J. L., \& Paller, K. A. (2010b). What makes recognition without awareness appear to be elusive? Strategic factors that influence the accuracy of guesses. Learning $\&$ Memory, 17, 460-468. 
Voss, J. L., Schendan, H. E., \& Paller, K. A. (2010). Finding meaning in novel geometric shapes influences electrophysiological correlates of repetition and dissociates perceptual and conceptual priming. Neuroimage, 49, 2879-2889.

Wiggs, C. L., \& Martin, A. (1998). Properties and mechanisms of perceptual priming. Current Opinion in Neurobiology, 8, 227-233.

Zago, L., Fenske, M. J., Aminoff, E., \& Bar, M. (2005). The rise and fall of priming: How visual exposure shapes cortical representation of objects. Cerebral Cortex, 15, $1655-1665$. 